Accepted for publication in Psychoneuroendocrinology

*Note: This is an uncorrected version of an author's manuscript accepted for publication.*Copyediting, typesetting, and review of the resulting proofs will be undertaken on this manuscript before final publication. During production and pre-press, errors may be discovered that could affect the content.

\title{
Polarity- and valence-dependent effects of prefrontal transcranial direct current stimulation on heart rate variability and salivary cortisol
}

Andre R. Brunoni ${ }^{1,2}$; Marie-Anne Vanderhasselt ${ }^{3}$; Paulo S. Boggio ${ }^{4}$; Felipe Fregni ${ }^{5}$; Eduardo Miranda Dantas ${ }^{6}$; José G. Mill ${ }^{6}$; Paulo A. Lotufo ${ }^{1}$; Isabela M. Benseñor ${ }^{1}$

(1) Clinical Research Center, University Hospital, University of São Paulo, Brazil.

(2) Department of Neurosciences and Behavior, Institute of Psychology, University of São Paulo, Brazil.

(3) Department of Experimental Clinical and Health Psychology, Ghent University, Belgium.

(4) Cognitive Neuroscience Laboratory and Developmental Disorders Program, Centre for Health and Biological Sciences, Mackenzie Presbyterian University, Sao Paulo, Brazil.

(5) Laboratory of Neuromodulation, Spaulding Rehabilitation Hospital, Harvard Medical School, Boston (MA), USA.

(6) Federal University of Espírito Santo, Vitória, Brazil

Running title: Neuroendocrine effects of tDCS

Corresponding author: Andre R Brunoni, MD

Clinical Research Center, University Hospital, University of São Paulo

Av. Prof Lineu Prestes 2565, 3o andar, CEP 05508-000, São Paulo (SP), Brazil

Tel +551130919241; e-mail: brunoni@usp.br 


\begin{abstract}
Recent evidence has supported the notion that the hypothalamic-pituitary-adrenal (HPA) and the sympatho-adreno-medullary (SAM) systems are modulated by cortical structures such as the prefrontal cortex. This top-down modulation may play a major role in the neuroendocrine changes associated with stressful events. We aimed to investigate further this hypothesis by modulating directly prefrontal cortex excitability using transcranial direct current stimulation (tDCS) - a non-invasive, neuromodulatory tool that induces polaritydependent changes in cortical excitability - and measuring effects on salivary cortisol and heart rate variability as proxies of the HPA and SAM systems. Twenty healthy participants with no clinical and neuropsychiatric conditions were randomized to receive bifrontal tDCS (left anodal/right cathodal or left cathodal/right anodal) or sham stimulation, in a withinsubject design. During each stimulation session, after a resting period, subjects were shown images with neutral or negative valence. Our findings showed that excitability enhancing left anodal tDCS induced a decrease in cortisol levels. This effect is more pronounced during emotional negative stimuli. Moreover, vagal activity was higher during left anodal tDCS and emotional negative stimuli, as compared to sham stimulation and neutral images. We also observed an association between higher mood scores, higher vagal activation and lower cortisol levels for anodal stimulation. Subjective mood and anxiety evaluation revealed no specific changes after stimulation. Our findings suggest that tDCS induced transient, polarity specific modulatory top-down effects with anodal tDCS leading to a down-regulation of HPA and SAM systems. Further research using tDCS and neuroendocrine markers should explore the mechanisms of stress regulation in healthy and clinical samples.
\end{abstract}




\section{Introduction}

The dorsolateral prefrontal cortex (DLPFC) has been associated with a wide range of cognitive functions from selective attention, working memory, decision-making including emotional behavior and empathy (Cerqueira et al., 2008, Damasio, 2000) to regulation of mood and anxiety (Davidson et al., 2002). In fact, one important role of DLPFC is in processing and regulating stressful, emotional responses - for instance, DLPFC shows activation when voluntarily suppressing sadness (Levesque et al., 2003) and co-activation with the amygdala during emotion reappraisal (Banks et al., 2007). Immediately following a stressful event, subcortical areas (such as the amygdala, hypothalamus and brainstem monoaminergic nuclei) trigger strong (albeit relatively unspecific) neuroendocrine responses, notably the activation of the sympatho-adreno-medullary system (SAM) and the hypothalamic-pituitary-adrenal (HPA) axis, thereby increasing noradrenaline and cortisol levels. In this context, further DLPFC neural processing may either lead to a top-down regulation, ultimately inhibiting SAM and HPA activity or, conversely, subcortical areas might down-regulate cortical activity, with subsequent increasing in SAM and HPA functioning. This latter, "bottom-up" pattern (amygdala hyperactivity / DLPFC hypoactivity) is observed in mood and anxiety disorders, both during resting state and cognitive/challenging neuroimaging and functional studies (Quide et al., 2012, Britton et al., 2011, Drevets et al., 2008). In addition, analogous to other cortical structures, DLPFC has lateralized properties: while the left side is associated with parasympathetic activity, positive emotional processing and approach behavior; the right PFC is associated with sympathetic activity, negative emotional processing and withdrawal behavior (Cerqueira et al., 2008). Indeed, mood and anxiety disorders are associated with hypoactivity of the left DLPFC (Davidson et al., 2002).

Nevertheless, most research in the field is correlational, and experimental manipulation is necessary to increase our insight in the causal relationship between cortical functioning, emotional regulation and specific stress responses. In this context, transcranial direct current stimulation (tDCS) is one interesting tool to investigate such processes. TDCS is a neuromodulatory technique that consists in applying a direct electric current through electrodes positioned over the scalp to induce local and secondarily distance neuroplasticity. Although tDCS induced current is weak, it is able to reach the neuronal tissue and induce polarization-shifts on cortical excitability (Nitsche et al., 2008, Brunoni et al., 2011c). Remarkably, anodal stimulation generally facilitates cortical activity, whereas cathodal tDCS has opposite effects (Brunoni et al., 2011c). In fact, studies have shown polarity-dependent 
tDCS effects for several cortical areas, including occipital, sensory, motor and prefrontal cortex areas (Utz et al., 2010).

Therefore, considering the importance of DLPFC in emotional processing and stress responses in healthy individuals as well as in neuropsychiatric disorders, we sought to test whether tDCS of DLPFC regulates emotional processes and stress responses by measuring heart rate variability (HRV) and salivary cortisol levels as they reflect SAM and HPA functioning (Marques et al., 2010). Specifically, while the former assesses the relative influences of sympathetic and vagal branches over heart beat-to-beat activity (ESC, 1996); the latter increases as a stress response to negative and unpleasant stimuli (Hellhammer et al., 2009).

In this sham-controlled study, two different montages were used: left anodal / right cathodal stimulation (thus increasing left DLPFC cortical excitability and an opposite effect on the right DLPFC) and, conversely, right anodal / left cathodal stimulation (thus inducing a contrary effect). Emotional responses were induced using images of negative valence. Based on the current knowledge in the lateralized DLFPC processing of emotional information and its top-down inhibitory properties, we hypothesized that the former montage would be associated with decreased and the latter with increased SAM and HPA activity, respectively, as indexed by HRV and cortisol measures. In despite of gaining mechanistic insights of DLPFC and tDCS functioning, this study is also important to measure safety - i.e., whether tDCS could exert hazardous effects in autonomic activity, as anecdotally reported (Redfearn et al., 1964) - and to also support the exploration of tDCS for potential clinical gains - e.g., regulation of blood pressure (Cogiamanian et al., 2010) and stress response.

\section{Methods}

\section{Subjects}

Twenty healthy participants with no psychiatric or clinical conditions were included. Their mean age was $24.9(S D=3.8)$ years and three were male. They were recruited among university students at the University of São Paulo, Brazil. All volunteers gave written informed consent and the local Ethics Committee approved the study. No financial compensation was given. We screened 31 subjects. Of them, a trained psychiatrist excluded those with prior and current psychiatric disorders by a psychiatrist using the M.I.N.I. questionnaire (Sheehan et al., 1998) and the Beck Depression Inventory (BDI) (Gorenstein et al., 2000) - those with scores higher than 8 were excluded $(n=4$, with acute depressive 
episode and $\mathrm{n}=1$ with generalized anxiety disorder). In addition, we performed biochemistry, anthropometric and EKG evaluations as to exclude those with potential cardiovascular conditions ( $\mathrm{n}=1$, with sinus bradycardia and first-degree atrioventricular block). We also excluded participants who perform intense physical activity $(n=3$, university students who competed in academic leagues), as this was related to changes in HRV measures in a prior tDCS study (Montenegro et al., 2011). Moreover, women in the luteal phase had their participation postponed to the subsequent follicular phase as to minimize individual cortisol variability (Kajantie and Phillips, 2006). For the same reason, women with irregular menses were excluded ( $\mathrm{n}=2$, due to polycystic ovary syndrome and possible pregnancy).

\section{Design}

A randomized, sham-controlled, within-subjects design was used. Subjects were randomized in a counterbalanced order to receive all stimulation conditions (left anodal, left cathodal or sham). To avoid carry-over effects between sessions, subjects had to attend to the research center in three different days, with a minimal interval of 48 hours between them. All sessions occurred between 10 a.m. and noon. Subjects were asked to avoid drinking coffee and smoking cigarettes $24 \mathrm{~h}$ prior to the sessions. None of them was under medication use.

Each session lasted 40 minutes and was divided in four consecutive phases, each of which lasting ten minutes: in the first phase, the HRV and tDCS electrodes were positioned on the participant, who was then sat in a comfortable chair. TDCS was started during the last three minutes of this first phase. In the second, third and four phases we showed images of neutral, negative and neutral valences, respectively; they were presented in a 15 -inch LCD monitor for six seconds, with a 500ms interval between them. The images were shown for eight minutes and in the last two minutes of phases III and IV there was an interval in which salivary cortisol was collected (in phases I and II no assessments were done; phase I was a "resting", initial period; phase II was designed as a training, standardization period). The current emotional state of the subject was assessed using the visual analogue scales (VAS) for mood and anxiety. Each image was shown only once for a given participant. These images were selected from the International Affective Picture System (IAPS) database, being rated according to their level of valence and arousal (Lang et al., 1993). We selected pictures with mean arousal values $<4$ and mean valence ratings between 2.5-3.9 (negative) and 5-6 (neutral). (Figure 1)

(Figure 1)

TDCS was delivered by a battery-driven stimulator with two rubber electrodes placed in $5 \times 7 \mathrm{~cm}$ saline-soaked sponges. Electrodes were positioned over the F3 or F4 areas 
according to the 10/20 EEG International System that corresponds to the regions over the left and right dorsolateral prefrontal cortex (DLPFC), respectively. For the left anodal stimulation, the anode was at F3 and the cathode at F4; for the left cathodal stimulation, the cathode was at F3 and the anode at F4. This montage has been used in several studies (Ferrucci et al., 2009, Brunoni et al., 2011b). For the verum tDCS we used a $1.5 \mathrm{~mA}$ current, for the sham condition the device was turned off after 30 seconds of stimulation, a sham method proved to be reliable for blinding purposes (Gandiga et al., 2006). We also assessed blinding asking subjects to guess whether they had received active or sham stimulation.

\section{Assessments}

After each phase we evaluated subjective affective changes asking the participants to grade their current emotional state according to the visual analogue scales (VAS) for mood and anxiety: the participants were asked to describe how they felt "at the moment" by indicating on horizontal, 10-cm lines, on a range from "absolutely not" to "very much". We also performed the Affect and Negative Affective Schedule (PANAS) and State-Trait Anxiety Inventory (STAI) questionnaires at the beginning of the study. They both present Portuguesevalidated (Gorenstein et al., 2000, Galinha and Pais-Ribeiro, 2005) versions and are frequently used questionnaires to clinically evaluate mood and anxiety. These variables were categorized in two levels (low vs. high) accordingly to the $25^{\text {th }}$ (for positive affect) and the $75^{\text {th }}$ percentile (for negative affect, state anxiety and trait anxiety) of the scores. We used these cut-off points specifically aiming to assess those with increased psychopathological symptoms. Finally, adverse events were assessed through a specific questionnaire (Brunoni et al., 2011a).

Electrocardiographic evaluations were carried out in a calm room in controlled temperature. A digital electrocardiograph (Micromed, sampling rate of $250 \mathrm{~Hz}$ ) was used to acquire the records. The electrodes were placed on the limbs, and the signals were recorded from the higher R-wave amplitude lead (usually D2). We used Wincardio 4.4 to generate the beat-to-beat R-R interval series. The artifacts and ectopic beats were corrected by the spline cubic interpolation method. HRV analysis was performed in the frequency domain for the low- (LF-HRV, 0.04-0.15 Hz) and high-frequency (HF-HRV, 0.15-0.4 Hz) bands that are related with sympathetic and vagal activity, respectively (ESC, 1996). Power spectral analysis was performed by the autoregressive method and model order was set at 16 (Boardman et al., 2002). Importantly, although there are several HRV measures (including time, frequency and non-linear analyses) we analyzed only LF- and HF-HRV as they are especially useful when assessing the contrast between sympathetic vs. parasympathetic activity during emotional and stressful tasks (Thayer et al., 2011). Also, considering that common HRV software usually 
extracts 25 measures (Johnsen Lind et al., 2011), we used only the total spectral power $\left(\mathrm{ms}^{2}\right)$ of LF- and HF-HRV measures as to not inflate type I error.

Saliva samples were collected using Salivettes with an insert containing a sterile polyester swab, yielding a clear and particle-free sample. They were used according to the instructions provided by the manufacturer. The salivettes were centrifuged at $1500 \mathrm{rpm}$ for 15 minutes, and the filtrates were stored frozen $(-20 \mathrm{C})$ until analysis that was performed with standard ELISA kits from DIAsource ImunoAssays SA, Belgium. Before analysis, the sample were thawed and individually recentrifuged.

Analysis

All statistical analyses were performed using Stata 12 (Statacorp, TX). For the dependent variables we used the mood and anxiety VAS scores as well as the natural logarithmic values of HF-HRV, LF-HRV and the square root values of cortisol (the data were normalized as the identity values were non-parametric), each of which being analyzed separately (i.e., we analyzed only one variable at a time). As to assess carry-over effects we included the term order of stimulation in a separate model.

The independent variables were polarity (left anodal; left cathodal and sham), valence (neutral vs. negative) and the interaction between them. Therefore, our main model was a repeated-measures, within-subject ANOVA. Importantly, we used the Greenhouse-Geisser correction whether the assumptions of sphericity were not met.

Whether the valence $\mathrm{x}$ polarity interaction was significant $(p \leq 0.05)$, further models were explored in which positive affect, negative affect, anxiety-state and anxiety-trait from the PANAS and STAI scales were individually analyzed as between-subjects factors. In addition, we also modeled VAS scores as covariates to explore whether changes in mood or anxiety were associated with the dependent variable. Since this was an exploratory study, we did not adjust $p$ levels for multiple comparisons.

\section{Results}

No tDCS adverse events were reported and participants did not correctly guess whether they were receiving active or sham stimulation $\left(\chi^{2}=1.4 ; p=0.23\right)$.

(Table 1)

(Table 2) 
For HF-HRV, we found no significant main effects for polarity $(\mathrm{F}=0.13 ; \mathrm{p}=0.88)$ and valence $(F=1.58 ; p=0.23)$. However, a significant polarity $\mathrm{x}$ valence interaction was found $(\mathrm{F}=2.99 ; \mathrm{p}=0.05)$. Post-hoc analyses for this interaction showed that HF-HRV values were significantly different $(\mathrm{F}=4.9 ; \mathrm{p}=0.04)$ for left anodal vs. sham stimulation when comparing images of negative vs. neutral valence - in fact, HF-HRV values were higher for negative vs. neutral images during left anodal stimulation, and vice-versa for left cathodal stimulation (Tables 1 and 2). The effect on HRV was specific for the HF as for LF-HRV measures, the repeated-measures ANOVA showed no significant effects for polarity $(F=0.41 ; p=0.66)$, valence $(F=2.66 ; p=0.12)$ and the interaction between them $(F=0.86 ; p=0.42)$. (Figure 2)

(Figure 2)

\section{Salivary cortisol}

Significant effects were found for valence $(\mathrm{F}=10.1 ; \mathrm{p}<0.01)$ (with higher cortisol levels for negative images) and the valence $x$ polarity interaction $(\mathrm{F}=3.1 ; \mathrm{p}=0.04)$ - here, the post-hoc analysis showed significant differences for left anodal vs. sham stimulation according to image valence; specifically, that for left anodal stimulation cortisol values were lower and almost similar for both valences whereas they were higher for sham stimulation, and even higher for negative images (Tables 1 and 2). For the main effect of polarity alone, however, the repeated-measures ANOVA model showed a trend $(F=2.39 ; \mathrm{p}=0.1)$ for a significant main effect for polarity, with lower values for left anodal and higher values for left cathodal stimulation; supporting the notion that polarity effects are also specific for the valence. (Figure 3)

(Figure 3)

Finally, as to further explore the changing in cortisol levels, we estimated a new dependent variable that was the percentage based on the difference of values between active and sham, divided by the values observed during sham stimulation as to normalize values according to sham stimulation, i.e,

Change in cortisol $=100 \times($ Active - Sham $) /($ Sham $)$ 
Using these values as a new dependent variable, we performed a new model in which polarity had only two levels (anodal vs. cathodal). In this model, we observed a trend for valence effects $(F=2.68 ; p=0.11)$ and significant effects for polarity $(F=8.64 ; p<0.01)$ and for the interaction of polarity vs. valence $(\mathrm{F}=4.6 ; \mathrm{p}=0.04)$. Specifically, for polarity effects, we observed that anodal stimulation was associated with $5.3 \%$ lower $(\mathrm{SE}=15)$ whereas cathodal stimulation with $64.3 \%$ higher ( $\mathrm{SE}=24$ ) cortisol levels. Considering the polarity vs. valence interaction, we found that anodal stimulation was associated with cortisol levels $12 \%$ lower $(\mathrm{SE}=12)$ for negative pictures and virtually no changes $(+1 \%)$ for neutral pictures. Conversely, cathodal stimulation was associated with 106\% $(\mathrm{SE}=39)$ and $22.6 \%(\mathrm{SE}=18)$ higher cortisol levels for negative and neutral pictures, respectively.

\section{Relationship between cortisol and HF-HRV}

Since we identified a polarity $\mathrm{x}$ valence interaction for both HF-HRV and cortisol, we sought to explore whether these changes were correlated. In a post-hoc analysis, we performed regression models for each polarity (anodal, cathodal and sham) using changes in cortisol and HF-HRV as variables. We found that these variables were inversely related $(\beta=-$ $0.16, p=0.03$ ) after anodal stimulation (i.e., higher cortisol values were related with lower HFHRF values), but not during cathodal and sham stimulation ( $\mathrm{p}=0.64$ and $\mathrm{p}=0.24$, respectively).

\section{VAS for mood and anxiety}

As expected, VAS mood values were significantly lower for negative than neutral pictures $(\mathrm{F}=15.63 ; p<0.01)$. Likewise, VAS anxiety values were significantly lower for neutral than negative pictures $(\mathrm{F}=22.6 ; p<0.01)$. For both mood and anxiety VAS values there were no significant main effects for polarity $(\mathrm{F}=1.14 ; p=0.33$ and $\mathrm{F}=1.9 ; 0.16$, respectively). We also did not observe significant effects on either $\operatorname{mood}(\mathrm{F}=0.18 ; p=0.83)$ or anxiety $(\mathrm{F}=1.56 ; 0.22)$ for the polarity vs. valence interaction (Tables 1 and 2$)$. In other words, the repeated-measures ANOVA showed, for subjective mood and anxiety assessments, significant changes for valence but not for polarity and the interaction between them.

We also used mood and anxiety VAS scores as covariates in the analyses that had a significant valence $x$ polarity interaction (i.e., for cortisol and HF-HRV). The variable VAS mood was found to be significant when cortisol $(\beta=-0.21, p=0.03)$ and HF-HRV $(\beta=0.09$, $\mathrm{p}=0.04$ ) were the dependent variables, suggesting that higher levels of cortisol and lower values of HF-HRV were associated with lower mood ratings. The variable VAS anxiety was 
not significantly associated for both cortisol and HF-HRV models ( $\mathrm{p}=0.44$ and $\mathrm{p}=0.33$, respectively).

Analysis of baseline assessments

The repeated-measures ANOVA models that presented significant polarity $\mathrm{x}$ valence interactions (i.e. HF-HRV and salivary cortisol) were further explored by successively adding the following between-subjects factors: positive affect, negative affect, anxiety-state and anxiety-trait - all categorized in high vs. low levels. However, we observed no significant interactions for these factors with the term polarity $\mathrm{x}$ valence.

Carry-over effects

To analyze carry-over effects, we added the term order of stimulation in the model. This term was not significant for any of the dependent variables ( $p>0.05$ for all analyses).

\section{Discussion}

The main finding of our study is that one single session of brain stimulation over the DLPFC changed HRV and salivary cortisol values according to tDCS polarity and the valence of imagery. Specifically, when subjects were presented with negative images, left anodal tDCS is associated with lower cortisol levels and higher HRV (HF-HRV values) as compared to sham stimulation. In addition, left cathodal tDCS, when compared specifically against left anodal tDCS, presented higher cortisol levels - this difference was more noticeable during negative imagery. To our knowledge, this is the first study assessing the emotional-triggering neuroendocrine responses associated with modulation of DLPFC excitability by transcranial direct current stimulation. We further discuss the observed changes in cortisol levels, HRV measures and subjective affective assessments.

To our knowledge, no previous study investigated top-down modulation using tDCS on salivary cortisol. Although Raimundo et al. (2011) evaluated cortisol changes after tDCS finding no specific changes - their results were based on a sub-sample analysis of blood cortisol levels. Interestingly, previous, single-session, sham-controlled, fast rTMS studies in healthy women showed no modulatory effects on the HPA axis after left or right DLPFC (Baeken et al., 2009b), although right DLPFC rTMS effects were observed, in another sample 
of healthy women, when controlling for baseline, state anxiety (Baeken et al., 2011). Other studies showed that fast rTMS over the left DLPFC decreased salivary cortisol levels in patients with depression (Baeken et al., 2009a) and bulimia (Claudino et al., 2010). The potent, polarity-dependent tDCS effects on the HPA axis observed in our study might be explained by the simultaneous bifrontal modulation, since previous models have suggested that HPA activity decreases or increases according to left and right DLPFC activity, respectively (Cerqueira et al., 2008). In addition, such effects were bolder when presenting negative valence imagery, demonstrating that they specifically demanded greater dorsolateral prefrontal cortical processing, thus amplifying subsequent tDCS effects (in contrast with neutral images that demand no specific, lateralized affective processing). In fact, tDCS was able to modulate the effects of images on salivary cortisol. This finding goes in agreement with tDCS as this technique is associated with modulation - but not induction - of spontaneous neuronal firing (Bindman et al., 1964).

For HRV, there were two previous studies investigating tDCS effects on HRV. These studies showed mixed findings. Vandermeeren et al. (2010) applied anodal, cathodal or sham tDCS in 30 healthy volunteers, finding no specific effects on HRV. In this study, the active reference was over the midline frontal cortex and an extra-cephalic reference was used. Conversely, Montenegro et al. (2011) applied anodal tDCS over the left temporal area (reference electrode: contra-lateral supraorbital area), observing an increase in HF-HRV and a decrease in LF-HRV values. However, this effect was observed only in athletic (as compared to non-athletic) subjects who were professional road race cyclists and, therefore, they do not constitute a fully representative sample. In our study, we found no main polarity effects on LF- and HF-HRV measures; nevertheless, a higher vagal activity was observed for left anodal tDCS during negative valence imagery. Here, the same framework applied for cortisol changing holds: left DLPFC activity was increased during anodal stimulation, thereby increasing the influence of the parasympathetic branch on the heartbeat - in fact, this effect was significant only during negative valence imagery that demands greater and specific DLPFC processing. However, we did not observe specific effects for the LF-HRV measures. In fact, it should be underscored that LF is not a "pure" marker: although described as a sympathetic marker, LF might reflect vagal activity as well (ESC, 1996). Therefore, we cannot definitely exclude sympathetic tDCS modulatory effects - further studies could use other techniques (e.g., skin conductance) to investigate this topic.

Regarding subjective emotional assessment (VAS scales), we observed no specific tDCS effects. Remarkably, a seminal study performed by Lippold \& Redfearn (1964) suggested a potent, single-session, polarity-dependent tDCS effect, observing that subjects were quieter after cathodal and more agitated after anodal stimulation; recent studies 
generally showed no affective changes after one session of tDCS in healthy subjects. For instance, using a similar bifrontal tDCS montage than ours, Plazier et al. (2011) did not observe profound mood changes in healthy subjects. Also, Koenigs et al. (2009) tried to replicate Lippold \& Redfearn's previous study using a similar design, although with more complete mood assessments. They observed no significant mood changes. Remarkably, they also evaluated mood and skin conductance changes after arousing IAPS pictures and tDCS, observing no interaction effects. Nevertheless, two previous studies assessed the effects of tDCS with images that were either unpleasant (Boggio et al., 2009) or had negative valence (Pena-Gomez et al., 2011), showing significant interactions between them. The discrepant findings compared to our study can be possibly explained by an underpowered analysis, as we collected data only after one set of images whereas these studies assessed VAS after each image. In fact, assessing subjective mood changes after continued, repetitive exposition of negative images could have allowed an emotional regulation for them - i.e., after the initial, impacting images that triggered emotional responses, the subject could have coped (i.e. cognitively reappraised) the subsequent imagery, thus leading to an overall less important net effect. Nonetheless, we opted for this design (instead of consecutively randomizing images with difference valences) as our primary aim was to assess the physiological HRV and cortisol responses rather than actively assessed their mood feelings towards the imagery.

Interestingly, positive mood was inversely and directly associated with cortisol and HF-HRV, respectively, throughout the experiment, i.e., higher mood ratings were related with lower cortisol and higher HF-HRV values. In addition, HF-HRV and cortisol values were inversely related during anodal stimulation. These findings corroborate the hypothesis of anodal tDCS effects on the left DLPFC and top-down modulation of subcortical structures (indexed by cortisol and HRV).

Of note, we observed no association for cathodal stimulation and HRV, and no association for HF-HRV and cortisol during cathodal stimulation. Also, cathodal stimulation did not increase cortisol levels as compared to sham. This might suggest that this type of stimulation is less potent than anodal tDCS in inducing SAM and HPA modulation, at least in healthy subjects who present adequate resilience to negative emotional stimuli. One hypothesis is that, in such samples, it might be easier to enhance stress resilience through anodal DLPFC stimulation than decrease it - possibly because other cortical mechanisms can play DLPFC role in top-down modulation and/or their amygdala is less prone to marked activation. Future studies could investigate whether these findings would be similar in patients with anxiety and depressive disorders.

\section{Limitations}


One limitation of our study is the relatively low spatial resolution of tDCS (Brunoni et al., 2011c). Therefore, other brain regions besides the DLPFC could also have been modulated. Since adjacent brain areas are also involved in affective processing, we cannot determine to which extent a possible adjacent modulation could have influenced the results (Wager et al., 2003). For instance, the ventromedial prefrontal cortex and orbitofrontal cortex are localized immediately below the DLPFC and are associated with emotional/affective processing (Koenigs and Grafman, 2009) - in fact, the activation of the ventromedial prefrontal cortex predicts antidepressant response of rTMS (Kito et al., 2012). Thus, the interpretation of our findings should also consider that the modulation of these areas could have occurred, either directly or indirectly, considering the connections of the DLPFC with them - and, hence, that the observed effects could be also explained by their hypo/hyperactivation. Interestingly, Fregni et al. (2005) showed that moving the electrodes from DLPFC $5 \mathrm{~cm}$ back to the primary motor cortex abolished tDCS effects on working memory, suggesting at least certain spatial resolution for tDCS. Technological advancement now allows fMRI studies during active tDCS, which might clarify to which extent areas beyond the DLPFC are activated during its stimulation.

Another limitation is that we did not explore the effects of tDCS on positive valence imagery, which could have been interesting as to further investigate cortical-subcortical processing. However, we chose to focus on negative valence imagery, as they tend to elicit stronger psychophysiological responses (Bradley et al., 2001). Finally, we did not randomize the block order, which could have influenced the results - we opted for having the same order as to maximize the contrast between negative and neutral images (as phase II always showed pictures of neutral valence).

\section{Conclusion}

To conclude, our results suggest that cortisol and HRV values - markers of autonomous and neuroendocrine systems - change after one single-session of non-invasive brain stimulation over the DLPFC during negative visual stimuli. Such changes vary and depend on tDCS polarity and the affective salience on imagery. We found that left anodal DLPFC stimulation was associated with lower cortisol levels and with higher HF-HRV measures as compared to sham stimulation. When anodal vs. cathodal tDCS were specifically compared, cortisol levels increased with cathodal and decreased with anodal stimulation, these effects being more pronounced during visual negative stimuli. Taken together, our findings suggest that tDCS is able to induce transient, modulatory top-down effects by showing that modulation of DLPFC excitability can also down-regulate the HPA and SAM systems. This can be an interesting framework for using tDCS in mood and anxiety disorders, 
in which such systems are dysfunctional. Our findings also support the safety of tDCS. Therefore, our findings give additional evidence for the specific relationship between DLPFC activity and HPA and SAM regulation and thus encourage further research using tDCS and neuroendocrine markers to explore the mechanisms of affective processing and top-down regulation in healthy and clinical samples.

\section{References}

BAEKEN, C., DE RAEDT, R., LEYMAN, L., SCHIETTECATTE, J., KAUFMAN, L., POPPE, K., VANDERHASSELT, M. A., ANCKAERT, E. \& BOSSUYT, A. 2009a. The impact of one HF-rTMS session on mood and salivary cortisol in treatment resistant unipolar melancholic depressed patients. Journal of affective disorders, 113, 100-8.

BAEKEN, C., DE RAEDT, R., LEYMAN, L., SCHIETTECATTE, J., POPPE, K., KAUFMAN, L., HAES, M., VANDERHASSELT, M. A., ANCKAERT, E. \& D'HAENEN, H. 2009b. The impact of one session of HF-rTMS on salivary cortisol in healthy female subjects. The world journal of biological psychiatry : the official journal of the World Federation of Societies of Biological Psychiatry, 10, 586-90.

BAEKEN, C., VANDERHASSELT, M. A. \& DE RAEDT, R. 2011. Baseline 'state anxiety' influences HPA-axis sensitivity to one sham-controlled HF-rTMS session applied to the right dorsolateral prefrontal cortex. Psychoneuroendocrinology, 36, 60-7.

BANKS, S. J., EDDY, K. T., ANGSTADT, M., NATHAN, P. J. \& PHAN, K. L. 2007. Amygdalafrontal connectivity during emotion regulation. Social cognitive and affective neuroscience, 2, 303-12.

BINDMAN, L. J., LIPPOLD, O. C. \& REDFEARN, J. W. 1964. The Action of Brief Polarizing Currents on the Cerebral Cortex of the Rat (1) During Current Flow and (2) in the Production of Long-Lasting after-Effects. J Physiol, 172, 369-82.

BOARDMAN, A., SCHLINDWEIN, F. S., ROCHA, A. P. \& LEITE, A. 2002. A study on the optimum order of autoregressive models for heart rate variability. Physiological measurement, $23,325-36$.

BOGGIO, P. S., ZAGHI, S. \& FREGNI, F. 2009. Modulation of emotions associated with images of human pain using anodal transcranial direct current stimulation (tDCS). Neuropsychologia, 47, 212-7.

BRADLEY, M. M., CODISPOTI, M., CUTHBERT, B. N. \& LANG, P. J. 2001. Emotion and motivation I: defensive and appetitive reactions in picture processing. Emotion, 1, 276-98.

BRITTON, J. C., LISSEK, S., GRILLON, C., NORCROSS, M. A. \& PINE, D. S. 2011. Development of anxiety: the role of threat appraisal and fear learning. Depression and anxiety, 28, 5-17.

BRUNONI, A. R., AMADERA, J., BERBEL, B., VOLZ, M. S., RIZZERIO, B. G. \& FREGNI, F. 2011a. A systematic review on reporting and assessment of adverse effects associated with 
transcranial direct current stimulation. The International Journal of

Neuropsychopharmacology, 1-13.

BRUNONI, A. R., FERRUCCI, R., BORTOLOMASI, M., VERGARI, M., TADINI, L., BOGGIO, P. S., GIACOPUZZI, M., BARBIERI, S. \& PRIORI, A. 2011b. Transcranial direct current stimulation (tDCS) in unipolar vs. bipolar depressive disorder. Prog Neuropsychopharmacol Biol Psychiatry, 35, 96-101.

BRUNONI, A. R., NITSCHE, M. A., BOLOGNINI, N., BIKSON, M., WAGNER, T., MERABET, L., EDWARDS, D. J., VALERO-CABRE, A., ROTENBERG, A., PASCUAL-LEONE, A., FERRUCCI, R., PRIORI, A., BOGGIO, P. S. \& FREGNI, F. 2011c. Clinical research with transcranial direct current stimulation (tDCS): Challenges and future directions. BRAIN STIMULATION.

CERQUEIRA, J. J., ALMEIDA, O. F. \& SOUSA, N. 2008. The stressed prefrontal cortex. Left? Right! Brain, behavior, and immunity, 22, 630-8.

CLAUDINO, A. M., VAN DEN EYNDE, F., STAHL, D., DEW, T., ANDIAPPAN, M., KALTHOFF, J., SCHMIDT, U. \& CAMPBELL, I. C. 2010. Repetitive transcranial magnetic stimulation reduces cortisol concentrations in bulimic disorders. Psychological medicine, 1-8.

COGIAMANIAN, F., BRUNONI, A. R., BOGGiO, P. S., FREGNi, F., CIOCCA, M. \& PRIORI, A. 2010. Non-invasive brain stimulation for the management of arterial hypertension. Med Hypotheses, 74, 332-6.

DAMASIO, A. R. 2000. Eighth C.U. Ariens Kappers Lecture. The fabric of the mind: a neurobiological perspective. Progress in brain research, 126, 457-67.

DAVIDSON, R. J., PIZZAGALLI, D., NITSCHKE, J. B. \& PUTNAM, K. 2002. Depression: perspectives from affective neuroscience. Annual review of psychology, 53, 545-74.

DREVETS, W. C., PRICE, J. L. \& FUREY, M. L. 2008. Brain structural and functional abnormalities in mood disorders: implications for neurocircuitry models of depression. Brain structure \& function, 213, 93-118.

ESC 1996. Heart rate variability. Standards of measurement, physiological interpretation, and clinical use. Task Force of the European Society of Cardiology and the North American Society of Pacing and Electrophysiology. Eur Heart J, 17, 354-81.

FERRUCCI, R., BORTOLOMASI, M., VERGARI, M., TADINI, L., SALVORO, B., GIACOPUZZI, M., BARBIERI, S. \& PRIORI, A. 2009. Transcranial direct current stimulation in severe, drug-resistant major depression. J Affect Disord, 118, 215-9.

FREGNI, F., BOGGIO, P. S., NITSCHE, M., BERMPOHL, F., ANTAL, A., FEREDOES, E., MARCOLIN, M. A., RIGONATTI, S. P., SILVA, M. T., PAULUS, W. \& PASCUALLEONE, A. 2005. Anodal transcranial direct current stimulation of prefrontal cortex enhances working memory. Exp Brain Res, 166, 23-30.

GALINHA, I. O. \& PAIS-RIBEIRO, J. L. 2005. Contribuição para o estudo da versão portuguesa da Positive and Negative Affect Schedule (PANAS): II - Estudo psicométrico (*). Análise Psicológica, 2, 219-227. 
GANDIGA, P. C., HUMMEL, F. C. \& COHEN, L. G. 2006. Transcranial DC stimulation (tDCS): a tool for double-blind sham-controlled clinical studies in brain stimulation. Clin Neurophysiol, $117,845-50$.

GORENSTEIN, C., ANDRADE, L. H. S. G. \& ZUARDI, A. W. 2000. Escalas de Avaliação Clínica em Psiquiatria e Psicofarmacologia, Sao Paulo.

HELLHAMMER, D. H., WUST, S. \& KUDIELKA, B. M. 2009. Salivary cortisol as a biomarker in stress research. Psychoneuroendocrinology, 34, 163-71.

JOHNSEN LIND, A., HELGE JOHNSEN, B., HILL, L. K., SOLLERS III, J. J. \& THAYER, J. F. 2011. A user-friendly application for the extraction of kubios hrv output to an optimal format for statistical analysis - biomed 2011. Biomedical sciences instrumentation, 47, 35-40.

KAJANTIE, E. \& PHILLIPS, D. I. 2006. The effects of sex and hormonal status on the physiological response to acute psychosocial stress. Psychoneuroendocrinology, 31, 151-78.

KITO, S., HASEGAWA, T. \& KOGA, Y. 2012. Cerebral blood flow in the ventromedial prefrontal cortex correlates with treatment response to low-frequency right prefrontal repetitive transcranial magnetic stimulation in the treatment of depression. Psychiatry and clinical neurosciences, 66, 138-45.

KOENIGS, M. \& GRAFMAN, J. 2009. The functional neuroanatomy of depression: distinct roles for ventromedial and dorsolateral prefrontal cortex. Behav Brain Res, 201, 239-43.

KOENIGS, M., UKUEBERUWA, D., CAMPION, P., GRAFMAN, J. \& WASSERMANN, E. 2009. Bilateral frontal transcranial direct current stimulation: Failure to replicate classic findings in healthy subjects. Clin Neurophysiol, 120, 80-4.

LANG, P. J., GREENWALD, M. K., BRADLEY, M. M. \& HAMM, A. O. 1993. Looking at pictures: affective, facial, visceral, and behavioral reactions. Psychophysiology, 30, 261-73.

LEVESQUE, J., EUGENE, F., JOANETTE, Y., PAQUETTE, V., MENSOUR, B., BEAUDOIN, G., LEROUX, J. M., BOURGOUIN, P. \& BEAUREGARD, M. 2003. Neural circuitry underlying voluntary suppression of sadness. Biological psychiatry, 53, 502-10.

LIPPOLD, O. C. \& REDFEARN, J. W. 1964. Mental Changes Resulting from the Passage of Small Direct Currents through the Human Brain. The British Journal of Psychiatry, 110, 768-72.

MARQUES, A. H., SILVERMAN, M. N. \& STERNBERG, E. M. 2010. Evaluation of stress systems by applying noninvasive methodologies: measurements of neuroimmune biomarkers in the sweat, heart rate variability and salivary cortisol. Neuroimmunomodulation, 17, 205-8.

MONTENEGRO, R. A., FARINATTI PDE, T., FONTES, E. B., SOARES, P. P., CUNHA, F. A., GURGEL, J. L., PORTO, F., CYRINO, E. S. \& OKANO, A. H. 2011. Transcranial direct current stimulation influences the cardiac autonomic nervous control. Neuroscience Letters, 497, 32-6.

NITSCHE, M. A., COHEN, L. G., WASSERMANN, E. M., PRIORI, A., LANG, N., ANTAL, A., PAUluS, W., HUMMEL, F., BOGGIO, P. S., FREGNI, F. \& PASCUAL-LEONE, A. 2008. Transcranial direct current stimulation: State of the art 2008. Brain Stimul, 1, 206-23. 
PENA-GOMEZ, C., VIDAL-PINEIRO, D., CLEMENTE, I. C., PASCUAL-LEONE, A. \&

BARTRES-FAZ, D. 2011. Down-regulation of negative emotional processing by transcranial direct current stimulation: effects of personality characteristics. PLoS One, 6, e22812.

PLAZIER, M., JOOS, K., VANNESTE, S., OST, J. \& DE RIDDER, D. 2011. Bifrontal and bioccipital transcranial direct current stimulation (tDCS) does not induce mood changes in healthy volunteers: a placebo controlled study. BRAIN STIMULATION.

QUIDE, Y., WITTEVEEN, A. B., EL-HAGE, W., VELTMAN, D. J. \& OLFF, M. 2012. Differences between effects of psychological versus pharmacological treatments on functional and morphological brain alterations in anxiety disorders and major depressive disorder: a systematic review. Neuroscience and biobehavioral reviews, 36, 626-44.

RAIMUNDO, R. J., URIBE, C. E. \& BRASIL-NETO, J. P. 2011. Lack of clinically detectable acute changes on autonomic or thermoregulatory functions in healthy subjects after transcranial direct current stimulation (tDCS). BRAIN STIMULATION.

REDFEARN, J. W., LIPPOLD, O. C. \& COSTAIN, R. 1964. A Preliminary Account of the Clinical Effects of Polarizing the Brain in Certain Psychiatric Disorders. Br J Psychiatry, 110, 773-85.

SHEEHAN, D. V., LECRUBIER, Y., SHEEHAN, K. H., AMORIM, P., JANAVS, J., WEILLER, E., HERGUETA, T., BAKER, R. \& DUNBAR, G. C. 1998. The Mini-International Neuropsychiatric Interview (M.I.N.I.): the development and validation of a structured diagnostic psychiatric interview for DSM-IV and ICD-10. J Clin Psychiatry, 59 Suppl 20, 2233; quiz 34-57.

THAYER, J. F., AHS, F., FREDRIKSON, M., SOLLERS, J. J., 3RD \& WAGER, T. D. 2011. A metaanalysis of heart rate variability and neuroimaging studies: Implications for heart rate variability as a marker of stress and health. Neuroscience and biobehavioral reviews.

UTZ, K. S., DIMOVA, V., OPPENLANDER, K. \& KERKHOFF, G. 2010. Electrified minds: Transcranial direct current stimulation (tDCS) and Galvanic Vestibular Stimulation (GVS) as methods of non-invasive brain stimulation in neuropsychology-A review of current data and future implications. Neuropsychologia, 48, 2789-810.

VANDERMEEREN, Y., JAMART, J. \& OSSEMANN, M. 2010. Effect of tDCS with an extracephalic reference electrode on cardio-respiratory and autonomic functions. BMC Neuroscience, 11, 38.

WAGER, T. D., PHAN, K. L., LIBERZON, I. \& TAYLOR, S. F. 2003. Valence, gender, and lateralization of functional brain anatomy in emotion: a meta-analysis of findings from neuroimaging. Neuroimage, 19, 513-31. 
Table 1. Dependent variables values during transcranial direct current stimulation and negative and neutral valence imagery.

Table 2. Repeated-measures ANOVA results for the interaction between the main outcomes and the independent variables.

Figure 1. Study design. Each session was composed by four consecutive phases, each of which lasting approximately 10 minutes: in the first phase the subject just remained sat, with eyes-open and electrodes positioned. In the second phase, transcranial direct current stimulation (tDCS) was started and neutral valence images were showed. In the third and fourth phases images of negative and neutral valences were showed, respectively. Heart rate variability, VAS and salivary cortisol were collected and analyzed during the $3^{\text {rd }}$ and $4^{\text {th }}$ phases. Each patient attended to three sessions in a randomized order, as to receive left anodal / right cathodal, left cathodal / right anodal or sham stimulation.

Figure 2. HF-HRV according to polarity and valence. The graph shows highfrequency heart rate variability (HF-HRV) values (natural logarithmic transformation) according to the polarity (anodal, cathodal or sham) and the valence of imagery (negative and neutral). As depicted, vagal activation was higher during anodal stimulation and negative imagery. Bars represent standard errors (SE).

Figure 3. Salivary cortisol according to polarity and valence. The graph shows high-frequency salivary cortisol values (square root transformation) according to the polarity (anodal, cathodal or sham) and the valence of imagery (negative and neutral). As depicted, cortisol levels were lower for anodal stimulation, especially during negative imagery. Bars represent standard errors (SE). 